\begin{tabular}{|l|l|l|l|}
\hline CHIRONOMUS & VOI.1 & NO.1 & PLON.JULI 1967 \\
\hline
\end{tabular}

\title{
CHIRONOMUS
}

MITTEILUNGEN AUS DER CHIRONOMIDENKUNDE

\section{Zum Anfang}

Jeder, der mit Chironomiden zu tun hat, weiß, daß unsere Kenntnis dieser Dipteren-Familie, insbesondere ihre systematische Erfassung, kaum den Stand erreicht hat, der in den meisten anderen Insektengruppen schon vor etwa 100 Jahren überwunden war. Die schon beschriebenen Arten sind teilweise so schlecht durchgearbeitet, daß es z.B. nicht möglich ist, mit der vorhandenen Literatur die Chironomiden Europas zu bestimmen; von anderen Teilen der Welt sind sie meist nur fragmentarisch bekannt.

In Gegensatz zu unserem lückenhaften Wissen. über die Taxonomie steht die große Bedeutung, die die Chironomiden innerhalb vieler Forschungsrichtungen in steigendem Maße einnehmen; z.B. Limnologie-Okologie, Zoogeographie, Genetik, Cytologie, Physiologie, Biochemie etc. Obwohl man dem Trend der Zeit entsprechend heute systematische Forschungsarbeit im allgemeinen kaum fördert, mehren sich erfreulicherweise von Jahr zu Jahr die Wissenschaftler, die mit Chironomiden arbeiten. Dennoch ist ihre Anzahl, gemessen an der zu bewältigenden Aufgabe noch immer sehr gering. Die Arbeit mit Chironomiden ist nicht nur durch die große Zahl der Arten mit ihrer ungewöhnlich uniformen Morphologie, sondern ebenso dadurch erschwert, daß zur Definierung der Art die Metamorphose berücksichtigt werden muß.

Es ist dringend notwendig, alle vorhandenen Kräfte zu einer wirksamen Zusammenarbeit anzuregen, damit die Chironomidenkunde die Versäumnisse vergangener Jahrzehnte aufzuholen vermag. Das 1. Internationale Chironomidensymposium 1964 kann als der erste und erfolgreiche Schritt zur Verwirklichung dieses Zieles gewertet werd $n$. Das 2 . Internationale Chironomidensymposium wird demnächst, vom 7. - 9. August, auf Einladung von Herrn Prof. PALMEN in Helsinki stattfinden.

Neben den weiterhin im Abstand von einigen Jahren geplanten Symposien erscheint es angebracht, in der Zwischenzeit die fachlichmkollegialen und freundschaftichen Kontakte durch ein Mitteilungsblatt weiter zu pflegen, um so die Zusammenarbeit noch wirksamer gestalten zu können.

Es ist gedacht, mehrmals im Jahr den "Chironomus" herauszubringen. Er soll u.a. zur Verbreitung aktueller Nachrichten aus der Chironomiden- 
kunde dienen, über Arbeitsvorhaben einzelner Kollegen informieren, Austausch von Material erleichtern und Adressen vermitteln. Es ist ferner an einen Referatedienst über die neuere Literatur, insbesondere der Grenzgebiete der Chironomidentaxonomie, gedacht. Das Gelingen unseres Vorhabens wird von der Mitarbeit der an der Chironomidenforschung interessierten Kollegen abhängen. In diesem Sinne bitten wir um Ihre Anregung und Hilfe.

\section{E.J. Fittkau, F. Reiss}

\section{Chironomidenforschung in Plön}

Auf Wunsch des Geschäftsführenden Direktors des Max-Planck-Institutes für Limnologie (vormals Hydrobiologische Anstalt der Max-PlanckGesellschaft), Prof. H.SIOLI, soll die von THIENEMANN begründete Tradition der Chironomidenforschung in Plön wieler intensiv weitergepflegt werken, nachdem sie mit dem Tode von Prof. A. ThIEN 0. HARNISCH und der Pensionierung von Prof. Fr. LENZ zum Stagnieren gekommen war. In den Jahren $1960-1963$ und 1965 hielt sich Dr. E.J. FITTKAU im Amazonasgebiet auf und konnte sich nur in begrenztem Maße mit Chironomiden beschäftigen. In den letzten Jahren hat FITTKAU mit der Bearbeitung seiner umfangreichen neotropischen Chironomidensammlung begonnen und inzwischen mehr als 300 Arten präpariert. Die weitere Sichtung des noch vorliegenden Materials wird noch viele Jahre benötigen. Um die europälsche chironomidenforschung nicht zu vernachlässigen und die vorhandene Sammlung durchzuarbeiten, wurde kürzlich Dr. F. REISS angestellt. Sein Hauptinteresse gilt $z$. $z$. der Gattung Micropsectra und den Chironomiden alpiner Seen. Das Erbe von Prof. 0. HARNISCH pflegt seit einigen Jahren mit atmungsphysiologischen Untersuchungen an Chir ronomus-Larven Frau Dr. I. PLATZER-SCHULTZ. Als Doktoranden von Prof. J. ILLIES, Fuldastation des Max-Planck-Institutes für Limnologie, arbeitet Herr HOFMANN über die Chironomiden der sedimente zweier Seen des Plöner Gebietes, die Herren LEHMANN und RINGE über die Chironomiden der Fulda. Neben den erwähnten Arbeiten wird mit Unierstützung aller in Frage kommenden Kollegen die Herausgabe einer Monographie der "Chironomiden Europas" vorbereitet.

Die Voraussetzungen für die Arbeit mit Chironomiden sind in Plön in jeder Hinsicht gut. In den Labors steht ausreichend platz und optisches. Gerät zur Verfügung. Die Chironımidenliteratur ist, dank der langjährigen Bemühungen THIENEMANNs, zumindest in taxonomisch-morphologischem Bereich ziemlich vollständig. Die Chironomidensammlung enthält in Dauerpräparaten und Alkoholmaterial etwa $600-800$ europäische Arten. In ihr sind enthalten die Coll. THIENEMANN und seiner Schüler, 
die Coll. LENZ und die Sammlungen von FITTKAU und REISS. (Die Coll. STRENZKE ist nach seinem Tode an das Senckenberg-Museum, Frankfurt, gekommen. Aus der Coll. THIENEMANN fehlten nur die Diamesini, die, entliehen an PAGAST, durch Kígseinwirkung vernichtet wurden. Besonders wertvoll ist die Sammlung durch die zahlreichen Metamorphosestadien, die vielfach zu verlorengegangenen Typen KIEFFERs und GOETGHEBUERs gehören. Umfangreiche Literatur- und Art-Karteien sind vorhanden bzw, werden aufgebaut.

Gäste haben jederzeit gut ausgeristete Arbeitsplätze zur Verfügung, um die gebotenen Möglichkeiten aușunutzen. Verschledentlich hat die Max-Planck-Gesellschaft dankenswerterweise devisenschwachen Kollegen Stipendien zur Verfugung gestellt, um ihnen einen Arbeitsaufenthalt zu ermöglichen.

\section{Anschriften von Fachkollegen}

Aufgefürt sind die uns $\mathrm{z} . \mathrm{Z}$. bekannten Kollegen, die in irgendeiner Weise mit Chironomiden arbeiten, Wir bitten um Mitteilung noch fehlender Adressen und um entsprechende Hinweise bel Anschriftenänderung, um die Liste In den folgenden Nummern verbessern und ergänzen zu können.

Argentinien

TORRES, Prof. Dr. B.A., Universidad Nacional de La Plata, Facultad de Ciencias Naturales y Museo, La Plata Australien

MARTIN, Dr.J., Zool. Dept., Univ. of Melbourne, Parkville, Victoria, Melbourne

Brasilien

OLIVEIRA, Dr. S.J. de, Inst. Oswaldo Cruz, C.P. 926, Rio de Jane Iro

Bulgarien

DIMITROV, Dr. M. , Stanzia po sladkovodno ribarstro, Plovdiv Canada

ACTON, Dr. A.B., Dept. of Zool., Univ. of Brit. Columbia, Vancouver 8 , Brit. Columbia

HYNES, Dr. R., Canada Dept. of Agricult, Central Exper. Farm, Room 1004 , K.W. Neatby Bldg., Ottawa, Ont.

REMPEL, Prof. Dr. I.G., Biology Dept., Univ. of Saskatchewan, Saskatoon, Sask.

SCUDDER, G. , Dept. of Zoology, Univ. Brit. Columbia, Vancouver 8, Brit. Columbia
VOCREROTH, Dr. I.R., Insect System, Sci. Service, Dept. of Agricult., ottawa, ont.

WENSLER, Dr. Rita J.D., Univ. of Saskatchewan, Dept. of Blology, Saskatoon, Sask. CSSR

ERTLOVA, Dr. Eva, Slovenská Akad. Vied. Sienkiewiczova 1 , Bratislava

HRABE, Prof. Dr. W., Zool. Ustav Masarykovy Iniv., Kotlarská 2, Brno

HRUSKA, V., Hydrobiol. Lab., CSAV, Vltavska 17, Praha 5-Simichov

LELLAK, Dr. J., Hydrobiologické a Ichthyologické Oddeleni, Zool. Ustavu Karlovy Univ., Vinicna 7, Praha II Dänemark

ANDERSEN, Dr. F.S., Statens Skadedyrlabor., Strandrejen 740 , Springforb $i / K o p e n h a g e n$

BERG, Prof.Dr. K. , Ferskvands-biol. Labor. Hillerød

DAH', mag.scient. J., Carlshфjvej 56 , Lyngby

JONASSON, P.M. , Freshwater Biol. Lab., Univ. of Copenhagen, Hillerød 


\section{Deutschland}

BEERMANN, Prof. Dr. W. , MaxPlanck-Inst.f. Biologie, Spemannstr. 34, 74 Tübingen

BRAUNITZER, Doz. Dr. G., MaxPlanck-Inst.f. Blochemie, Goethestr. 31, 8 München 15

CASPERS, Prof. Dr. H., Zool. Staatsinst., Hydrobiol. Abt., VonMelle-Park 10, 2 hamburg FITTKAU, Dr. E.J., Max-PlanckInst.f. Limnologie, 232 Plön GÖrZ, Dr. P., Zool. Inst. d. Univ., Katharinenstr. 20, 78 Freiburg

HENNIG, Prof. Dr. W., Staatl. Mus. f. Naturkunde, Zweigstelle Ludwigsburg, Arsenalplatz 3, 714 Ludwigsburg

HOFFRICHTER, 0., Zool. Inst, d. Univ., Katharinenstr. 20, 78 Freiburg

HOFMANN, W., Max-Planck-Inst . $f$. Limnologie, 232 Plön

KEYL, Doz. Dr. H.-G. , Max-PlanckInst. f. Meeresbiologie, Melanchthonstr. 36,74 Tübingen

LEHMANN, J., Limnolog. Flußstat. , Max-Planck-Inst. f. Limnologie, 6407 Schlitz/Hessen

LENZ, Prof. Dr. Fr., Prinzenstr.12, 232 Plön

MECHELKE, Prof. Dr. F., Inst, f. Allgem. Genetik, 7 StuttgartHohenhe im

MOELLER, Dr. J., Nds.-Forstl. Versuchsanst., Grätzelstr.2, 34 Göttingen

NEUMANN, Doz. Dr. D., Zool. Inst., Röntgenring 10, 87 Würzburg

PELliNG, Dr. C. , Max-Planck-Inst. f. Biologie, Spemannstr. 34, 74 Tübingen

PlatzER-SCHULTZ, Dr. I. , MaxPlanck-Inst. f. Limnologie, 232 Plön

REMMERT, Priv,-Doz. Dr. H, Z Zool. Inst., Hegewischstr. 3,23 Kiel

REISS, Dr. Fr. , Max-Planck-Inst. f. Limnologie, 232 Plön

RINGE, Fr. , Limnolog. Flußstat. Max-Planck-Inst. f. Limnologie, 6407 Schlitz/Hessen

SCHLEE, Dr. D. , Staatl. Mus, f. Naturkunde, Arsenalplatz 3, 714 Ludwigsburg

STEFFAN, Dez, Dr. A.W., II. Zool. Inst. d.Univ., Postfach 606 , $65 \mathrm{Mainz}$

TICHY, Dr. H., Max-Planck-Inst. f. Blologie, Spemannstr. 34 , 74 Tübingen
WULKER, Prof. Dr. H., Zool. Inst. d. Univ., Katharinenstr. 20, 78 Frej.burs Deutschland (DDR)

ALBRECHT, Dr. Marie-Luise, Jastrowerweg 21, 1 Beriin-Friedrichshagen

BARTHELMES, Dr. D., Inst. f. Binnenfischere1 DAL, Müggelseedamm 310, X 1162 BerlinFriedrichshagen

MOTHES, Dr: G. , Forschungsstelle f. Limnologie, Außenstelle Stechlinsee, $\dot{X} 1431$ Neuglobsow, Krs. Gransee

WUNDSCH, Prof. Dr. W.W. Rahnsdorfer-Str. 48, 1 Berlin-Friedrichshagen

Finniland

HIRVENOJA, Dr. M., Zool. Inst. d. Univ. Helsinki, P.-Rautatiekatu 13, Helsinki 10

LINDEBERG, Dr. B., Zool. Mus.d. Univ, Helsinki, P.-Rautatiekatu 13, Helsinki 10

PaLMen, Prof. Dr. E., Zool. Ingt. d. Univ., P.-Rautatiekatu 13, Ifelsinki 10

SYRJAMÄKI, Dr. J., Zool. Inst. d. Univ., P. -Rautatiekatu 13, Helsinki 10

Frankreich

DEJOUX, C., Centre O.R.S.T.O.M. de Fort-Lamy, Av, du Général Tilho, B.P. 65, Fort-Lamy, Tschad/Afrika

GoUIN, Dr. F., Mus. Zool., 29. Boulev. de la Victoire, strasbourg/Bas. Rhin

LAVILLE, H. , Univ, de Toulouse, Lab. de Zoologie, 118 Route Narbonne, 31 Toulouse

SERRA-TOSIO, B., Faculté des Sci. de Grenoble, B.P. 19, St. Martin d'Heres

TOURENQ, J.N., Lab. de Zoology de la Faculté des Sci, de Toulouse, Stat. Biol. de la Tour du Valat par le Sambuc (B, $\left.-d u-R_{\text {. }}\right)$

VAILLANT, Dr. F. , 26, Cours Berriat, Grenoble (Isère)

GroBbrittanien

BIRIETT, Dr, N.I., 3, Thorny Hills, Kendal/Westmorland

BRYCE, Dr. D., Dept. of Zoology, The Univ, , Reading

COE, Dr. R.L., Brit. Mus, (Nat. Hist.), Cromwell Road, London SW 7

EDWARDS, Dr. R.W., Water Pollut.Res. Labor., Stevenage, Herts 
FORD, Dr. J.B., Dept. of Agricult. and Forest Zool., Univ, of Nurth Wales, Bangor

FREEMAN, Dr. F., Brit. Mus. (Nat. Hist.), Cromwell Road, London SW 7

HALL, Dr. R.E., Dept. of Zoology The Univ., Southempton

HENSON, Dr. H. , Univ. of Leeds, Leeds

HINTON, Prof, Dr. K.E., Univ., Dept. of Zoology, Bristol

KARUNAKARAN, Dr. Letha, Zool. Dept., Univ. of Singapore, Bukit Timah Road, Singapore 10

MACAN, Dr. T.T., Freshwater Biol. Assoc., The Ferry House, Far Sawrey, Ambleside, Festmorland

MAITLAND, Dr. P.S., Dept. of Zool., Univ. of Glasgow

MORGAN, Dr. N.C., The Natur Conservancy, 12 Hope Terrace, Edinburgh 9

MUNDIE, Dr. J.H., Dept. of Zool., Univ, of Leicester, Leicester

PELHAM-CLINTON, Dr. E.C., Moredun Inst., Gilmerton, Edinburgh 9

WADDELL, Dr. A.B., Freshwater Fisheries Lab., Pitiochry, Marine Lab., Aberdeen Holland

KRUSEMAN, Dr. G. , Zool. Mus, , Afd. Entomologie, Zeeburgerdi jk 21, Amsterdam (0)

WEBER, Dr. R,E., Nederlands Inst. v. Onderzoek der Zee, Buitenhaven 27, Den Helder Irland

HUMPHRTES, Prof. Dr. C.F., Zool. Dept., Science Bldgs. Univ. College, Merrion street, Dublin Israel

KUGLE $\bar{R}, \overline{D r}$. J., Dept. of Zool., 155. Herzl Str., Tel-Aviv Univ., Tel-Aviv

Italien

MARCUZZI, Prof. Dr. G., Inst, di Zool., Padova

Japan

HASHIMOTO, Dr. H., Shimoda Marine Biolog. Stat., Tokyo Kyoiku Univ. Shimoda, Shizyoka Pref.

TOKUNAGA, M.Prof. Dr., Ent. Lab., Fac, of Agricult. Kyoto Prefectural Univ. Shímogamo, Kyoto Norwegen

SAETHER, Dr. O.A., Univ, of Oslo, Dept. of Limno?., Blindern-0sio 3 Polen

CZECZUGA, Dr. B., Zaklad Biol. Akad. Meycznef, U1. Kilinskiego 1 , Bialystok
KAJAK, Dr. Z., Inst. of Ecology, Dept. of Hydrobiol., Nowy Swiat 72, Warszawa

KOWNACKI, A., Zaklad Biologii Wod, Polska Akad. Nauk, ul. Slawskowska 17, Krakow

ROMANISZYN, Dr. W., Park Sienkicwicza, Lodz

ZIEBA, Dr. J., Zaklad, Biologii Wod, Polska Akad. Nauk, ul. Slawskowska 17, Krakow Rumänien

ALBU, Dr. Paula, Fac. de Sci., Splaiul Independentei $91-93$, Bukarest

BOTNARIUC, Prof. Dr. N., Fac. de Sc1. Nat., Splaiul Independentel 91-93, Bukarest

CANDEA, Dr. W. , Inst. de Biol. Anim, Acad, R,P.R., Splaiul Independentei 91-9.3, Bukarest Südafrika

HARRISON, Dr. A.D., Dept, of Zool. Univ. of Natal, P.0. Box 375 ,

Pietermaritzburg Schweden

BRUND IN, Prof. Dr. L., Naturhistoriska Riksmuseet, Ent. Avdeln., Stockholm 50

GRIMẢS, Dr. U., Zool. Inst. d. Univ. Cppsala

KURJK, A., Box 99, Messaure

NILSSON, Dr. N,-A, Zool. Inst. d. Univ., Uppsala

Schweiz

KROEGER, Doz. Dr. H, , Zool. Inst, d. E.T.H., Universitätsstr.2, Zürtch

Türke1

SENGUN, Prof. Dr. A., Zool. Inst. d. Univ., Istanbul UdSSR

ELBERG, Dr. K., Vabarüklik Sanitaar, Epidemiologiline Iaam, c/o Zool. Inst. Akad. Nauk ESSR, Vanemina 46 Tartu, ESSR

JABLONSKAJA, Dr. E.A., VNIRO Lab. of Hydrobiol., V. Krasnoselskaja 17, Moscow

KALUGINA, Dr. N.S., Mus, of Zool., Dept. of Entomol., Herzena VI, Moscow

KONSTANTINOW, Prof, Dr. A.S., Abt. Saratow d. Kaspi Filiale WNIORCH Ulitza Tschernischewsko:vo 162, Saratov

KORENEVA, Dr. T. A., State Univ. of Moscow, Biol.-Agricult. Facuity Len iskie Gory, Moscowv 234 
KRUGLOVA, Dr. V.M. , State Univ. of Rostov, Inst. of Biology 0lympiadovka, Rostov a Donu

LAVROVSKY, Dr. V.A., Medical College of the Town Saratow

LINEVISH, Dr. A.A., Skolni per., d. II, kv. I. g. Irkutsk

PANKRATÓVA, Dr. V.J., Inst. of Zool. AN USSR, Universitetskaja, Nabereznaja 1 , Leningrad 164

SHILOVA, Dr. A.I. , Jaroslavsko1 oblasti Nekouz, Biostanziz Akad. Nauk, Borok

SOKOLOVA, Dr. N.J., State Univ, of Moscow, Biol.Agricult. Fac. Leninskie Gory, Moscow V-234

TólP, Dr. O., Zool. Inst. Akad. Nauk ESSR, Vanemmina 36 , Tartu

ZVERELA, Dr. O.S., AN USSR Kom1 $g$. Syktyvkar, Kommunisticaskaja, 24 Filial AN USSR Ungarn

BERCZIK, Dr. A., Inst. Zoosystem. Univ., Puskin Utca 3, Budapest VIII

DEVAI, G. , Kossuth Lajos Tudományegyetem Allattani Intézete, Debrecen 10

ENTZ, Prof. Dr. B., Biol. Forschungsinst., Tihany

FERENCZ, Dr. Magdalena, System.Zool. Inst. of the Univ. at Szeged, Táncsics Mihály street $\mathrm{Nr}$ 2, Szeged USA

AUGENFELD, Prof. Dr. J., Univ, of Oklahoma, Dept. Zool., Norman Oklahoma 73069

BAY, Dr. E.-C., Dept, of Biol. Control, Univ, of Calif., Riverside, Calif. 92502

BECK, Dr. Elizabeth, Bureau of Sanitary Eng., Florida State Board of Health, p.o. Box 210, Jacksonville, Florida

BECK, Dr. W.M. Jr.

BERG, Prof. Dr. C.ó, Coronell Univ. Dept. of Entomol., Ithaca N.Y.

BUCK, Dr. J., National Inst. of Health, Cambridge Univ., Cambridge

BUSCEMI, Dr. Ph.A., Dept. of Biol. Sci., Univ, of Idaho, Moscow, I daho

CLEVER, U., Dept. of Biol. Scl., Purdue Univ., Lafayette, Indiana COOKE, Dr. H.G. Elizabeth City State Coll., Filizabeth City N.C.
CURRY, Prof. Dr. L.L., Biol. Dept. Box 55 C.M.C.E., Mt. Pleasant, Michlgan 48858

DARBY, Dr. R.E., Dept. Life Sci., Sacramento state College Sacramento, Calif.

DEEVEY, Dr. E.S. Jr., Osborn Zool. Labor., Yale Univ., New Haven, Conn.

DENDY, Dr. J.S., United Nations, U.S. Embassy, Apo 928 Postmaster, San Francisco, Calif.

FAGAN, Dr. E.B., Dept. Entomo1., Univ. Missouri, Columbia, Miss.

FROMMER, Dr. S., Dept. of E-tomol.; Univ, of Calif., Riverside, Calif. 92502

GRODHAUS, Dr. G., Dept. of Puhlic Health, State of Calif., 2151 Berkeley Way, Berkeley, Calif. 94704

GUYER, Dr. G.E., Dept. of Entomol. Michigan State Univ., East Lansing, Mich.

JAMBACK, Dr. H., The Univ, of the State of New York, The State Education Dept., Albany New York 12224

LAUFER, H. , Dept. of Zool. 8-117, Univ. of Connecticut, Storrs, Conn. 06268

NEFF, St. E., Dept. of Entomol., Cornell Untv: Ithaca N.Y.

PATTERSON, Dr. W.S., Project Director, P.O. Box 944, Winter Haven 33881 , Florida

PROVOST, Dr. M.W. , Florida state Board of Health, Ent. Res. Center, P.0. Box 308, Vero Beach, Florida

REED, E.B., Zool. Dept., Colorado State Univ., Ft. Colilins, Colorado

ROBACK, Dr. S.S., Acad of Nat, Scl. Dept. of Limnol., 19th and The Parkway, Philadelphia 3

STAHL, J., Southern Illinois Univ., Carbondale, Ill. 62901

SUBLETTE, Prof. Dr. J.E., Eastern New Mexico Univ., Box' 114, Portales, New Mexico

WIRTH, Prof. Dr. W.W., Ent. Res. Branch, Un. States Dept. of Agricult. Washington 25, D.C.

WHITSEL, Dr. R.H., San Mateo County, Mosquito Abatement Dis., 1350 North Carolan Ave., Burilingame/ Calif.

CHIRONOMUS-Mitteilungen aus der Chironomidenkunde. - Erscheint mehrmals jährlich. Herausgegeben von E.J. FITTKAU und F. REISS, Max-Planck-

Institut für Limnologie, Abt. Tropenökologie, 232 Plön, Postfach 165,
Deutschland (BRD). 